\title{
1 Materials for the Stem Cell Factories of the Future
}

3 Adam D. Celiz ${ }^{1}$, James G. W. Smith ${ }^{2}$, Robert Langer ${ }^{3}$, Daniel G. Anderson ${ }^{3}$, David A.

4 Barrett ${ }^{4}$, David A. Winkler ${ }^{5,6}$, Martyn C. Davies ${ }^{1}$, Lorraine E. Young ${ }^{2}$, Chris Denning ${ }^{2}$,

$5 \quad$ Morgan R. Alexander ${ }^{1}$

$6{ }^{1}$ Laboratory of Biophysics and Surface Analysis, School of Pharmacy, University of Nottingham, Nottingham, NG7 2RD, UK.

$7{ }^{2}$ Wolfson Centre for Stem Cells, Tissue Engineering and Modelling, Centre for Biomolecular Sciences, University of Nottingham,

8 Nottingham, NG7 2RD, UK. ${ }^{3}$ David H. Koch Institute for Integrative Cancer Research, Department of Chemical Engineering,

9 Institute for Medical Engineering and Science, Massachusetts Institute of Technology, Cambridge, MA02139, USA. ${ }^{4}$ School of

10 Pharmacy, University of Nottingham, Nottingham, NG7 2RD, UK. ${ }^{5}$ CSIRO Materials Science \& Engineering, Bag 10, Clayton

11 South MDC 3169, Australia. ${ }^{6}$ Monash Institute of Pharmaceutical Sciences, 399 Royal Parade, Parkville 3052 , Australia.

13 The materials community is now identifying polymeric substrates that could permit 14 translation of human pluripotent stem cells (hPSCs) from lab-based research to 15 industrial scale biomedicine. Well defined materials are required to allow cell banking 16 and to provide the raw material for reproducible differentiation into lineages for large 17 scale drug screening programs and clinical use, wherein $>1$ billion cells for each 18 patient are needed to replace losses during heart attack, multiple sclerosis and 19 diabetes. Producing this number of cells for one patient is challenging and a rethink is 20 needed to scalable technology with the potential to meet the needs of millions of 21 patients a year. Here we consider the role of materials discovery, an emerging area of 22 materials chemistry that is in a large part driven by the challenges posed by biologists to materials scientists ${ }^{1-4}$. 


\section{Evolution of stem cell culture}

3 The term human pluripotent stem cells (hPSCs) describes both human embryonic

4 stem cells (hESCs), typically derived from the inner cell mass of pre-implantation

5 embryos $^{5}$, and human induced pluripotent stem cells (hiPSCs) derived by epigenetic

6 reprogramming of somatic cells with stem cell-associated factors ${ }^{6}$. Because hPSCs

7 can self-renew for months, if not years, in culture and can be induced to differentiate

8 into all three germ layers, they provide immense potential for regenerative medicine

9 and drug development, as well as providing new in vitro models of genetic disease ${ }^{7}$.

10 However, for biomedical applications to be realized, defined culture conditions need

11 to be established to eliminate batch variability and xenogenic contaminants.

12 Furthermore, scalable culture systems are required ${ }^{8-9}$. For adherent culture systems,

13 scalability is often achieved by increasing the surface area of a growth substrate in T-

1475 flasks $\left(75 \mathrm{~cm}^{2}\right.$ growth area) without compromising biological performance as 15 measured by pluripotency markers.

16 Compared to a cell derived proteinaceous mixture such as Matrigel ${ }^{10}$, polymers are 17 reproducible and amenable to large scale manufacture, e.g. injection molding is 18 currently used to form tens of millions of tissue culture vessels per year. Consequently

19 there are significant efforts to identify synthetic strategies with the ultimate aim being 20 an inexpensive polymer that can be used off the shelf without pre-adsorption of 21 proteins or immobilization of other biomolecules such as peptides. Furthermore, a 22 fully synthetic growth substrate would be amenable to automated robotic cell culture, 23 paving the way for stem cell factories manufacturing billions of hPSCs suitable for 24 clinical use. 
1 This review describes the development of growth substrates for hPSC culture from

2 cell extracts to polymeric-derived materials and assesses the cost and scalability

3 issues associated with the most recent advances in hPSC with a particular focus on

$4 \quad$ materials discovery.

$7 \quad$ Feeder layers to support hPSC growth

9 Over the last 15 years, progress towards more precisely defined culture systems for 10 hPSCs has been made, summarized in Table 1. Initial reports of hPSC culture 11 employed feeder layers of mouse embryonic fibroblasts (MEFs) to support the self12 renewal of hPSCs. Feeder layers provide a source of extracellular matrix (ECM) 13 proteins and growth factors such as vitronectin, transforming growth factor $\beta$ (TGF- $\beta$ ) 14 and Laminin-511 that aid hPSC proliferation and self-renewal. In 1998, Thomson et 15 al. employed a MEF feeder layer to support hPSC growth in a medium of $80 \%$ 16 Dulbecco's modified Eagle's medium (DMEM) supplemented with 20\% fetal bovine 17 serum (FBS), $1 \mathrm{mM}$ glutamine, $0.1 \mathrm{mM} \beta$-mercaptoethanol and $1 \%$ nonessential amino 18 acids (NEAA). However, the use of non-human (xeno) feeder layers and animal19 derived serum such as FBS represent a potential source of pathogens such as 20 endogenous retroviruses and xeno epitopes such as nonhuman sialic acid, N21 glycolneuraminic acid (Neu5Gc) ${ }^{11}$. Factors such as Neu5Gc, a monosaccharide, can 22 induce an immune response upon transplantation of hPSCs cultured using xenogenic 23 methods and limit their use to in vitro applications. 


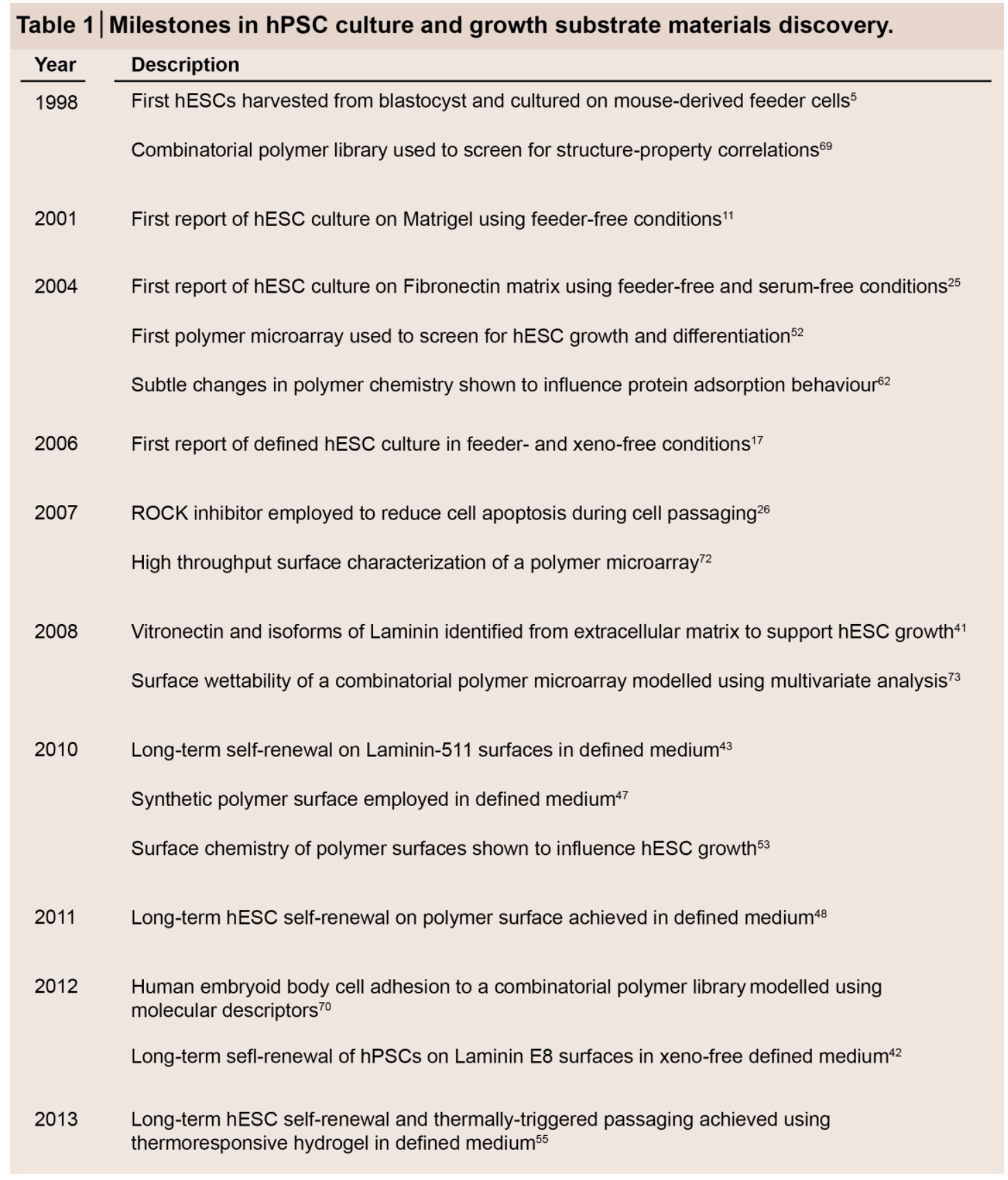

3 Feeder-free hPSC culture

4

5 To produce hPSCs that are safer and more useful in clinical applications, feeder-free

6 culture systems have been developed. The need to replace feeder layers with

7 alternative growth substrates has driven a huge research effort in the discovery of

8 materials that support the long-term self-renewal of hPSCs. Initial work on feeder-free 
1 systems in 2001 employed animal-derived growth substrates such as Matrigel in 2 combination with a MEF-conditioned medium $(\mathrm{MEF}-\mathrm{CM})^{10}$. Matrigel is harvested

3 from Engelbreth-Holm-Swarm (EHS) mouse sarcoma cells and consists of a complex

4 mixture of various extracellular matrix (ECM) proteins, proteoglycans and growth

5 factors $^{12}$. Unfortunately, culture systems consisting of undefined growth substrates

6 like Matrigel and MEF-CM exhibit batch-to-batch variability. These elements within

7 a culture system make understanding and controlling the cell-material interface

8 difficult, a prerequisite to developing a scalable and reproducible hPSC culture

9 system. Furthermore, batches of Matrigel have been contaminated with lactate

10 dehydrogenase-elevating virus (LDV). This highlights safety concerns with xenogenic

11 media components, although this particular pathogenic risk can be avoided with the 12 use of Geltrex, an undefined LDV-free growth substrate (Table 2) ${ }^{11,13-24}$. Although,

13 Matrigel can now also be purchased LDV-free.

14 Concerns over xenobiotic contamination have prompted the development and use of 15 serum-free media in combination with growth substrates containing recombinant 16 proteins $^{25}$. In 2004, Amit et. al. successfully demonstrated the self-renewal of I3, I6 17 and H9 hPSC lines on a fibronectin matrix using a serum replacement consisting of 18 various growth factors known to play a role in maintenance of pluripotency. Basic 19 fibroblast growth factor (bFGF), transforming growth factor $\beta 1$ (TGF $\beta 1$ ) and 20 leukaemia inhibitory factor (LIF) were tested in different mixtures. A combination of 21 TGF $\beta 1$ and bFGF with and without LIF was able to maintain pluripotency and retain 22 normal hPSC features on a human fibronectin growth substrate. However, growth 23 rates and cloning efficiencies of all combinations were inferior to MEF controls. 
Table 2| Commercialized growth substrates and culture media for hPSC culture.

\begin{tabular}{|c|c|c|c|c|c|c|}
\hline Component & Product & & & & & \\
\hline & & Defined & Synthetic & Xeno-free & Origin & Form \\
\hline \multirow{7}{*}{$\begin{array}{l}\text { Growth } \\
\text { substrate }\end{array}$} & Matrigel $^{\mathrm{TM}}, \mathrm{BD}$ Biosciences ${ }^{11}$ & $x$ & $\times$ & $\times$ & Cell extract & Gel \\
\hline & Geltrex $x^{T M}$, Invitrogen & $x$ & $x$ & $x$ & Cell extract & Gel \\
\hline & Synthemax ${ }^{\top M}$, Corning ${ }^{13}$ & $\checkmark$ & $\checkmark$ & $\checkmark$ & $\begin{array}{l}\text { Peptide-polymer } \\
\text { conjugate }\end{array}$ & $\begin{array}{l}\text { powder/coated } \\
\text { cultureware }\end{array}$ \\
\hline & StemAdhere ${ }^{T M}$, Primorigen Biosciences ${ }^{14}$ & $\checkmark$ & $\checkmark$ & $\checkmark$ & $\begin{array}{l}\text { Recombinant protein } \\
\text { (E-Cadherin) }\end{array}$ & Liquid \\
\hline & NunclonVita $^{T M}$, Thermo Scientific $^{15}$ & $\checkmark$ & $\checkmark$ & $\checkmark$ & $\begin{array}{l}\text { Plasma treated } \\
\text { polystyrene }\end{array}$ & Cultureware \\
\hline & CellStart ${ }^{\mathrm{TM}}$, Invitrogen ${ }^{16}$ & $\checkmark$ & $\checkmark$ & $\checkmark$ & $\begin{array}{l}\text { Humanized } \\
\text { protein mixture }\end{array}$ & Liquid \\
\hline & & Defined & Feeder-free & Xeno-free & Serum-free & \\
\hline \multirow{9}{*}{$\begin{array}{l}\text { Culture } \\
\text { medium }\end{array}$} & HEScGRO, Millipore & $\checkmark$ & $x$ & $\checkmark$ & $\checkmark$ & \\
\hline & mTeSR $1^{T M}$, STEMCELL Technologies ${ }^{17}$ & $\checkmark$ & $\checkmark$ & $x$ & $\sqrt{ }$ & \\
\hline & TeSR $^{T M} 2$, STEMCELL Technologies ${ }^{17}$ & $\checkmark$ & $\checkmark$ & $\checkmark$ & $\checkmark$ & \\
\hline & StemPro $^{\mathrm{TM}}$, Invitrogen ${ }^{18}$ & $\checkmark$ & $\checkmark$ & $x$ & $\checkmark$ & \\
\hline & NutriStem $^{\mathrm{TM}}$, STEMGENT $^{19}$ & $\checkmark$ & $\checkmark$ & $\checkmark$ & $\checkmark$ & \\
\hline & $\mathrm{E}^{\mathrm{TM}}, \mathrm{GIBCO}^{20}$ & $\checkmark$ & $\checkmark$ & $\checkmark$ & $\checkmark$ & \\
\hline & $\mathrm{XVIVO}^{\mathrm{TM}}$ 10, Lonza $^{21-22}$ & $\checkmark$ & $\checkmark$ & $\checkmark$ & $\checkmark$ & \\
\hline & $\operatorname{RegES}^{23}$ & $\checkmark$ & $x$ & $\checkmark$ & $\checkmark$ & \\
\hline & hESF $9^{24}$ & $\checkmark$ & $\checkmark$ & $\checkmark$ & $\sqrt{ }$ & \\
\hline
\end{tabular}

3 A major milestone in hPSC culture was the development of a defined culture medium

4 called TeSR 1 published in $2006^{17}$. The essential ingredients within the TeSR 1

5 medium were the proteins basic fibroblast growth factor (bFGF) and transforming

6 growth factor beta (TGF $\beta$ ), Lithium Chloride, $\gamma$-aminobutyric acid (GABA) and

7 pipecolic acid. Cell lines $\mathrm{H} 1$ and $\mathrm{H} 9$ were both shown to self-renew for more than 10

8 passages on a xeno-free growth substrate consisting of human collagen IV,

9 fibronectin, laminin and vitronectin. The derivation of two new hPSC lines, WA15

10 and WA16, was also achieved using TeSR1. However, WA15 became trisomic (three 
1 chromosomes as opposed to two) for chromosome 12 between 4 and 7 months in

2 culture. A variant of the TeSR1 culture cocktail has been commercialized as mTeSR1,

3 employing BSA and zebrafish bFGF, for a cheaper alternative to the xeno-free culture

4 medium. To further aid hPSC culture, supplements such as Rho-associated kinase

5 (ROCK) inhibitors have been employed to reduce dissociation-induced cell apoptosis

6 when working in defined medium ${ }^{26}$. However the impact of these inhibitors and their

7 long-term effects on hPSCs are yet to be understood.

8

9 High throughput screening has been used to discover small molecules that improve 10 hPSC survival and self-renewal, to improve hPSC culture methodology. In $2010 \mathrm{Xu}$ et

11 al. published a high throughput chemical screen of 50,000 synthetic compounds to 12 identify small molecules added to the culture media that promoted hPSC survival after 13 trypsin dissociation from a Matrigel substrate ${ }^{27}$. Thiazovivin (Tzv) and Pyrintegrin 14 (Ptn) were both found to dramatically increase cell survival versus DMSO controls. 15 High throughput screening of small molecules can rapidly identify essential 16 ingredients within current culture media employed in hPSC culture and be used to 17 reduce the number of components within the hPSC culture system (Box 1$)^{26-40}$. 18 Furthermore, this screening approach can identify novel culture medium supplements 19 to aid the survival and self-renewal of hPSCs in defined culture conditions. 


\begin{tabular}{|c|c|}
\hline $\begin{array}{l}\text { Improving current culture systems for the expansion } \\
\text { of hPSCs is essential before the full potential of } \\
\text { hPSCs can be realised in clinical applications. To be } \\
\text { routinely used in clinical applications, hPSCs would } \\
\text { ideally be produced in a good manufacturing practice } \\
\text { (GMP) grade culture system. Currently, the } \\
\text { definition of GMP in terms of hPSC technologies is } \\
\text { still being established as undefined culture systems } \\
\text { have entered phase } 1 \text { clinical trials. However, with } \\
\text { the advent of defined substrates, regulators will } \\
\text { ineveitably require all the components of a GMP } \\
\text { grade culture system to be xeno-free, fully defined, } \\
\text { and amenable to large scale production, ideally in an } \\
\text { automated process. GMP-grade hPSCs would have } \\
\text { to be fully characterised following large scale } \\
\text { production to ensure that normal karyotype, } \\
\text { proliferate rate, pluripotency-associated marker } \\
\text { profile and differentiation potential had been } \\
\text { maintained following long term culture within the } \\
\text { system. The success of hPSC culture systems to } \\
\text { produce such cells depends on controlling the } \\
\text { dynamic interactions that occur between the hPSCs, } \\
\text { the medium components and the growth substrate. } \\
\text { As such, there has been much recent investigation } \\
\text { into the development of defined synthetic substrates, } \\
\text { as well as defined synthetic medium supplements } \\
\text { that improve hPSC culture systems. } \\
\text { Traditional medium supplements most commonly } \\
\text { consist of biological molecules such as growth } \\
\text { factors and serum proteins to aid successful hPSC } \\
\text { culture. Recently, small molecule chemistries have } \\
\text { been shown to influence hPSC behaviour in culture, } \\
\text { including differentiation activity and reprogramming } \\
\text { potential. Importantly, there have been numerous } \\
\text { molecules reported to promote the survival and self- } \\
\text { renewal of hPSCs when added to the culture medium } \\
\text { (Table Bl). }\end{array}$ & $\begin{array}{l}\text { These molecules can manipulate hPSC activities by } \\
\text { the activation or inhibition of key molecules within } \\
\text { signalling pathways including MEK (PD0325901 and } \\
\text { PD98059) } \\
\text { 1077, and ROCK (Y27632, Thiazovivin, HA- } \\
\left.\text { PD173074) } \text {, }^{32} \text {, ERK (SC1) }\right)^{33} \text {, FGF (SU5402 and GSK3 (Bio, } \\
\text { CHIR99021, and Bisindolylmaleimide } 1 \text { i) } 28,34-35 \text {. As } \\
\text { such, small molecule chemistries hold great potential } \\
\text { as medium supplements in the development of fully } \\
\text { defined and cost-effective hPSC culture conditions } \\
\text { that are amenable to GMP scale-up. } \\
\text { Table B1 I Small molecule supplements for hPSC } \\
\text { survival and self-renewal. }\end{array}$ \\
\hline
\end{tabular}

3 Protein-based growth substrates

5 Following the identification of mixtures of ECM proteins as adsorbates required for

6 hPSC growth and self-renewal, effort was focused on identifying which proteins are

7 most effective with specific media. Miyazaki et al. identified the laminins (LN)-111, -

8332 and -511 as successful substrates for hPSC culture when used in combination

9 with MEF-CM ${ }^{41}$. The hPSC lines KhES-1 and KhES-3 were found to express

10 pluripotency markers after 10 passages and showed equal growth on LN-332 
1 compared to Matrigel at 72 hours. The utility of these substrates was attributed to

2 their high affinity for the $\alpha_{6} \beta_{1}$ integrin expressed on hPSCs. More recently, analogues

3 of these substrates employing laminin E8 fragments (functionally minimal forms of

4 laminin that can bind the $\alpha_{6} \beta_{1}$ integrin) have been shown to support hPSC self-

5 renewal in defined xeno-free medium for 10 passages $^{42}$. Laminin-332E8 and -511E8

6 surfaces were able to support the self-renewal of H9, HES3, KhES-1 (hESC lines) and

7 iPS(IMR90)-1, 253G1 (iPSC lines) in mTeSR1 and StemPro medium. Furthermore,

8 all cell lines displayed normal karyotype at passage 10. These simplified laminin

9 substrates were demonstrated to be successful for hPSC expansion at larger scale, 10 such as T-75 tissue culture polystyrene flasks.

11 In a similar study, Rodin et al. demonstrated the long-term growth of hPSCs on LN12511 coated plates for 20 passages over 4 months in chemically defined O3 medium 13 and xeno-free $\mathrm{H} 3$ medium (both variants of TeSR 1$)^{43}$. Furthermore, cells were able to 14 attach and migrate over/across the LN-511 coating creating continuous cell 15 monolayers due to their affinity for the $\alpha 6 \beta 1$ integrin. This phenomenon was thought 16 to have aided the long-term self-renewal of the hPSCs. However, passaging the cells 17 required physical removal as clumps from the LN-511 coated plates rather than as 18 single-cell suspensions. This method of passaging is compatible with automation and 19 limits the scalability of this culture system.

\section{$21 \quad$ Peptide-based growth substrates}

23 Following the use of substrates coated with protein and protein fragments to promote 24 hPSC adhesion, simplified substrates presenting specific peptide sequences have been 25 developed to identify specific interactions at the cell-material interface that mediate 
1 stem cell behaviour. Microarrays of laminin fragments were presented via self-

2 assembled monolayers (SAMs) by spotting 18 thiol functionalized peptides onto gold

3 slides to rapidly identify cell-binding potential ${ }^{44}$. H1 and H9 cells that attached and

4 proliferated after 6 days on certain laminin sequences displayed pluripotency markers

5 at similar levels to Matrigel controls. However, scale up from micro array spots and

6 expansion and long-term self-renewal on these surfaces was not demonstrated.

7 Furthermore, the MEF-CM used to culture the hPSC makes this a complex and ill-

8 defined system which will contain many proteins and other biomolecules in the media

9 solution and adsorbed to the surface.

11 Carboxylate-containing acrylate monomers immobilized on plasma treated 12 polystyrene plates, and subsequently conjugated to various RGD-containing short 13 peptide sequences through the $\mathrm{N}$-terminus, have been used to generate peptide14 acrylate surfaces (PAS) ${ }^{45}$. Of the six peptides employed, only two, bone sialoprotein 15 (BSP) and VN-derived peptide, supported hPSC attachment, suggesting that RGD 16 alone is a necessary but not sufficient binding motif. BSP- and VN-PAS were able to 17 demonstrate long-term self-renewal (>10 passages) and were scalable to $75 \mathrm{~cm}^{2}$ 18 flasks. High surface density of the supportive peptide was required to achieve growth 19 rates similar to Matrigel, with concentrations of BSP ranging from $0.75-1 \mathrm{mM}$ 20 yielding $6-9 \mathrm{pmol} / \mathrm{mm}^{2}$ peptide density. VN-PAS coated flasks seeded with hPSCs 21 and cultured for 4 days in defined medium showed uniform cell distributions, typical 22 morphology and expressed the pluripotency marker OCT4. This substrate has been 23 developed commercially and marketed as Synthemax (Table 2) ${ }^{13}$, however, the 24 biological components used in this substrate means that it is expensive (ca. \$100/T75 25 flask) compared to the widely used laboratory growth substrate Matrigel (ca. \$15/T75 
1 flask). Another example of a growth substrate that has been commercially developed

2 is StemAdhere ${ }^{14}$. This growth substrate employs a fusion protein of the $\operatorname{IgG} \mathrm{Fc}$

3 domain and E-cadherin, a $\mathrm{Ca}^{2+}$ dependent cell-cell adhesion molecule, which is

4 coated onto polystyrene plates. This substrate was able to support long-term culture of

5 H9 cells (90 passages) in mTeSR1 medium. Similarly to Synthemax, the recombinant

6 nature of StemAdhere increases the expense of using these substrates significantly.

7 The materials cost alone to produce 1 billion hPSCs (an approximation for a single

8 patient intervention) is estimated to be $c a$. $\$ 10,000$ and $\$ 15,000$ for Synthemax and

9 StemAdhere respectively (Table 3). This cost is likely to be prohibitive for cell

10 expansion for clinical and biomedical use and is considerably greater than that using

11 Matrigel, which is estimated to be $c a$. $\$ 1,500$ (excluding the cost of cells and media)

12 to produce 1 billion hPSCs. Phase 1 trials have been passed by regulators using

13 Matrigel as an expansion substrate, but the exact meaning of GMP is still evolving for

14 hPSC technologies and as more defined systems become available they are likely to

15 be insisted upon.

16 


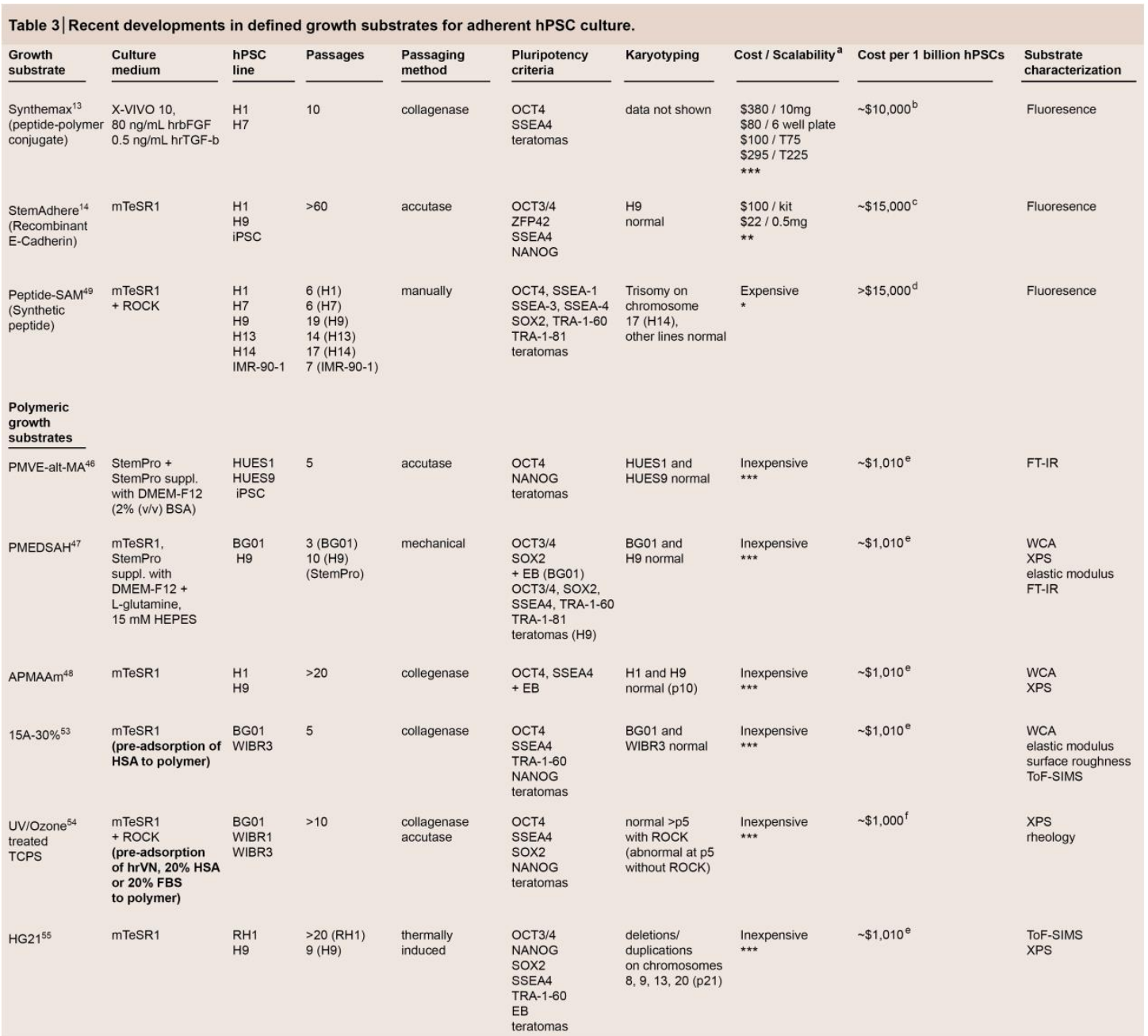

2 a Scalability determined by the ability to synthesize the material in large quantity for 3 manufacturing scale hPSC production in a cost-effective and timely manner, ${ }^{b}$ 4 estimated using $100 \mathrm{x}$ coated T75 flasks required to achieve 1 billion hPSCs, ${ }^{\mathrm{c}}$ 5 estimated by 150 kits required to coat a sufficient number of 6 well plates to achieve 1 6 billion hPSCs, ${ }^{\text {d }}$ estimated to be at least as expensive as a similar recombinant protein7 based substrate such as StemAdhere, ${ }^{\mathrm{e}}$ estimated by the cost of commercial monomers 8 of $c a$. $\$ 0.5 / \mathrm{g}$ and $50 \mathrm{mg}$ of dissolved polymer required to coat 350 T75 flasks, ${ }^{\mathrm{f}}$ 9 estimated by the cost of 350 T75 flasks.

\section{Polymer-based growth substrates}

13 Systems that we classify as scalable are those which can be used to produce billions 14 of cells in an economical and safe manner for many patients. For example, the 15 material cost of producing 1 billion hPSCs using a Matrigel coated substrate is $c a$. 
$1 \$ 1,500$, far cheaper than Synthemax and StemAdhere (Table 2). A benchmark in the

2 pharmaceutical industry to screen differentiated stem cells for drugs is to achieve a

3 cost of $<\$ 1$ per well (in a 96 well plate). This is not currently achievable for

4 pluripotent stem cell culture. The peptide-polymer derived substrates mentioned

5 previously are amenable to commercial development but ideally the substrate should

6 be fully synthetic using readily synthesized and cheap monomers.

7 Synthetic substrates that are polymer-based are generally inexpensive to manufacture

8 and have shown broad utility commercially. Brafman et. al. employed a polymer

9 microarray consisting of 91 commercially available and pre-synthesized polymers to

10 screen for hPSC attachment in StemPro medium ${ }^{46}$. A broad range of polymer

11 backbones and side chain functionalities were screened, including styrenes, acrylates 12 and acrylamides spotted onto acrylamide coated glass slides. Of the initial hits

13 identified by high OCT4 and NANOG expression using fluorescence microscopy to

14 identify pluripotency, one polymer, poly(methyl vinyl ether-alt-maleic anhydride)

15 (Figure 1d(ii)), was able to support hPSC attachment and self-renewal for >5

16 passages using StemPro medium. Scalable expansion of hPSCs on this polymeric

17 substrate was not demonstrated beyond cell expansion on polymer-coated slides.

19 Six unique acrylate-based surfaces have also been prepared via an ozone-activation of

20 tissue culture polystyrene (TCPS) and subsequent surface-initiated polymerization

21 with a range of acrylate monomers ${ }^{47}$. One of these materials, poly [2-

22 (methacryloyloxy)ethyl dimethyl-(3-sulfopropyl) ammonium hydroxide]

23 (PMEDSAH), was able to support the long-term culture of hPSC in serum-free

24 defined mTeSR1 medium (including protein supplement) (Figure 1d(iii)). H9 cells

25 were supported through 10 passages using StemPro medium, showed normal 
1 karyotype and expressed similar levels of pluripotency markers to cells cultured on

2 Matrigel. However, no scalability was demonstrated using this substrate material.

3 Irwin et. al. developed a methacrylamide based coating (Figure 1d(iv)) which was

4 grafted to TCPS dishes using a photoinitiated addition polymerization ${ }^{48}$. H1 and H9-

5 hOCT-pGZ hPSC lines were cultured in mTeSR1 medium for 10 passages,

6 maintained typical cell morphology and grew in colonies similar to Matrigel cultured

7 cells. Bovine serum albumin (BSA) was proposed to play a crucial role in hPSC

8 attachment achieved in the culture medium. Quartz crystal microbalance with

9 dissipation (QCM-D) experiments were used to identify BSA adsorption to the 10 growth substrate from the mTeSR1 medium.

11

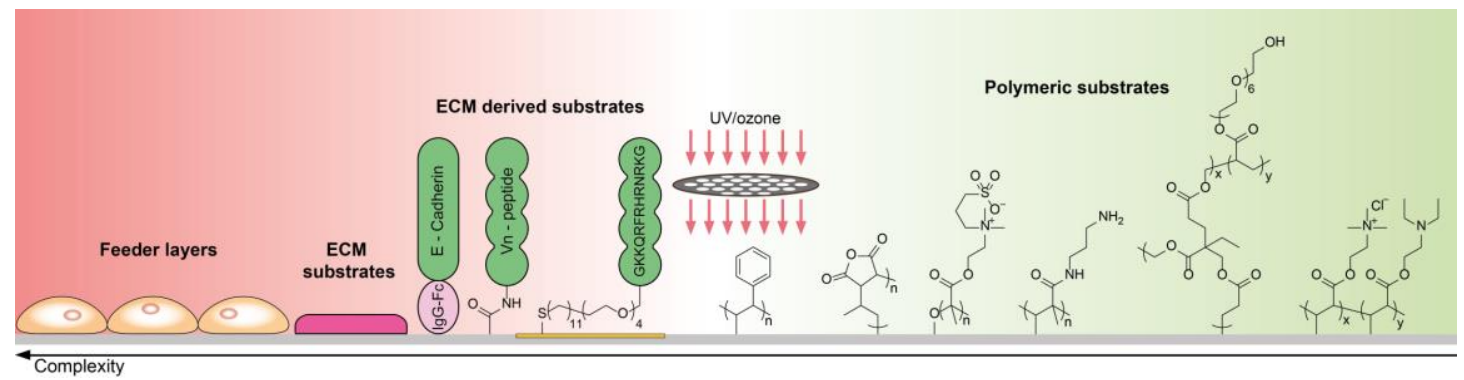

13 Figure 1 | The development of hPSC growth substrates. (a) Feeder layers of MEFs

14 to support cell adhesion and to condition the culture medium with ECM proteins to

15 facilitate hPSC self-renewal. (b) Surface coating with an undefined ECM protein-

16 containing mixture, e.g. Matrigel. (c) Functional epitopes of ECM components

17 immobilized to the surface to encourage hPSC attachment and self-renewal. (d)

18 Polymeric growth substrates to provide an environment to adsorb essential ECM

19 proteins from culture medium. 
1 High throughput materials discovery for stem cell culture

3 The defined growth substrates for adherent hPSC culture surveyed above have

4 limitations, and consequently the search for new materials for hPSC culture continues.

5 As we lack mechanistic understanding why cells respond to materials and media, high

6 throughput methodologies have been employed to screen as wide a combinatorial

7 chemical space as possible for materials supporting pluripotent cell number

8 expansion.

9 Surface modification strategies such as self-assembly have been used to present 10 molecules capable of binding to cell surface integrins with high spatial resolution

11 (Derda et $a l^{44}$ ). Arrays of peptide-substituted alkanethiols have been prepared as 12 self-assembled monolayers on gold surfaces ${ }^{49}$. The molecules screened included 13 peptides containing RGD and glycosaminoglycan binding epitopes, the most 14 successful of which being a heparin-binding peptide derived from vitronectin (VN) 15 (GKKQRFRHRNRKG). This peptide was able to support long-term self-renewal of 16 hPSCs at peptide densities of $0.5-25 \%$ (\% peptide-substituted alkane thiol in mixed 17 SAM monolayer) when combined with ROCK inhibitor or cyclic RGD peptide. This 18 peptide was used to functionalize glass and gold coated slides. Furthermore, 19 biotinylated-GKKQRFRHRNRKG was used to functionalize steptavidin-coated 20 TCPS dishes in a facile manner to reduce the cost of employing this peptide.

21 However, hPSC expansion was not demonstrated over large areas, e.g. a culture flask.

22 Pre-synthesized polymer libraries were printed as microarrays by Anderson et al. ${ }^{50}$ 23 employing the concept of combinatorial polymer libraries pioneered by Kohn et al in $241997^{51}$. For high throughput materials discovery, this has the limitation that polymer 25 synthesis is time consuming, reducing the diversity of such arrays and slowing the 
1 follow up of leads generated on subsequent arrays. Ideally, the evolution from the

2 initial screen to future generation arrays arises from hypotheses being formed from a

3 first generation array that are subsequently tested in generations that evolve rapidly

4 according to the results generated. On-slide synthesis of polymer microarrays was

5 achieved by Anderson et al. in $2004^{52}$, allowing rapid synthesis of acrylate polymers

6 by combinatorial mixing of liquid monomers printed onto a hydrogel coated slide

7 prior to UV photoinitiated free radical polymerization. A strength of polymer

8 microarrays is the ability to rapidly assess cell response to a large polymer library,

9 which when coupled with the ability to perform surface analysis of the library on the 10 array, allows cell-material surface interactions to be investigated rapidly ${ }^{3}$.

12 Subsequently, several groups have used polymer microarrays to screen surface

13 chemistries for hPSC attachment in a variety of culture media ${ }^{46,52,54}$. Mei et al.

14 screened a number of generations of polymer microarray, starting with a library of 15496 unique materials formed by mixing 16 acrylate 'major' monomers with 6 'minor' 16 monomers that were contact printed onto a poly(2-hydroxyethyl methacrylate) 17 (pHEMA) coated substrate and polymerized in-situ using UV irradiation ${ }^{53}$. Polymers 18 with potential as supports for pluripotent stem cells were identified by their ability to 19 support the clonal growth of BG01-Oct4-GFP ${ }^{+}$cells from very low initial seeding 20 densities over 7 days in MEF-CM (arrays pre-conditioned with FBS). High content

21 fluorescent microscopy was used to quantify cell response to the individual polymer 22 spots using OCT4 GFP. High throughput surface characterization of the microarray 23 was used to quantify the materials chemistry and properties such as wettability and 24 indentation elastic modulus, which were subsequently compared to cell performance 25 to identify the controlling surface factors. Colony-formation efficiency, a measure of 
1 the number of colonies formed at day 7 from the initial low cell attachment on day 1 ,

2 was used to quantify the performance of the hit substrates pre-adsorbed with ECM

3 proteins. Human vitronectin pre-adsorbed surfaces gave the highest colony formation

4 efficiency in mTESR1 medium maintaining pluripotency for 10 passages over 8

5 weeks on microarrays comprising the hit materials (monomer 9 and 15A), however,

6 only medium-term passaging ( $>5$ passages over 1 month) was reported on surfaces

7 pre-incubated with human serum albumin (HSA) and no scalability beyond

8 microarray spots was demonstrated for the hit materials (Figure 1d (v)).

9 A subsequent study used UV-ozone modification of polystyrene which maintained 10 pluripotency for $>10$ passages on surfaces conditioned with HSA or human $\mathrm{Vn}$ in 11 mTESR1 medium ${ }^{54}$. This growth substrate represents an attractive, cost-effective and 12 simple route amenable to scale up, although $\mathrm{Vn}$ is still required as a pre-adsorption 13 step for both approaches, which increases the cost of employing this culture system 14 (Figure 1d (i)).

15 More recently, Zhang et al. produced a microarray of 609 different thermoresponsive 16 polymers by ink-jet printing 18 acrylate and acrylamide monomers with a crosslinker 17 in various ratios ${ }^{55}$. The best performing material, an acrylate copolymer consisting of 18 trimethylammonium chloride and diethylamino side-groups (HG21), supported the 19 long-term self-renewal of RH1 cells (hPSC line) in mTeSR1 medium (>20 passages). 20 Karyotype analysis of the RH1 cells at passage 21 found chromosomal abnormalities.

21 This highlights the need for characterization of hPSCs at high passage number as 22 abnormalities can occur after several passages which render the hPSCs unusable for 23 clinical application.

24 The thermoresponsive nature of the copolymer hydrogel permitted cellular 25 detachment by reducing the culture medium temperature to $15^{\circ} \mathrm{C}$ for 30 minutes. This 
1 step may be useful in automated stem cell expansion systems. Growth rates of RH1

2 cells on hydrogel-coated coverslips were significantly lower than Matrigel controls;

3 RH1 cells took 8-10 days to reach $80 \%$ confluence on the hydrogel coating as

4 opposed 4-5 days on Matrigel in mTESR1 medium. Xeno-free culture medium was

5 not used and scalability of this growth substrate was not demonstrated beyond coating

6 of cover slips. The thermo switch of the gels was characterized by bulk rheology

7 measurements of materials on cover slips.

\section{Mechanism of cell response to surface cues}

12 Many research groups have directed their efforts towards understanding the effect of 13 materials on the regulation of stem cell behavior by designing substrates with 14 particular chemistries, compliances, topographies, or containing biologically relevant 15 moieties ${ }^{49,56-57}$. Cell adhesion molecules that govern cell-matrix and cell-cell 16 interactions play a crucial role in the long-term maintenance and self-renewal of 17 adhered hPSCs. The identification of cell surface integrins that can engage with 18 Matrigel $\left(\beta_{1}\right)$ and Vitronectin $\left(\alpha_{v} \beta_{3}\right.$ and $\left.\alpha_{v} \beta_{5}\right)$-coated substrates has enabled an 19 understanding of how hPSC pluripotency is maintained after they have adhered to 20 substrates ${ }^{42,53,58-59}$. Cell-cell interactions mediated by cadherins and their role towards

21 hPSC behavior has been extensively studied over recent years; see for example Li et

$22 \mathrm{al}$. for a recent review of this area ${ }^{60}$. Specifically, substrates presenting E-cadherin 23 have been commercialized as StemAdhere and have proven to be useful substrates for 24 hPSC expansion $^{14}$. 
1 Adsorption of individual proteins on polymeric substrates and the subsequent effect

2 upon cellular performance has been well studied; see for example Koenig et al. ${ }^{61}$ and

3 Weber et al. ${ }^{62}$. However, understanding which proteins adsorb from complex protein

4 containing media onto materials, and the conformation they adopt on adsorption to a

5 synthetic surface is still not yet possible, yet it is an essential element in gaining an

6 understanding of material performance in protein containing culture conditions. Such

7 knowledge will ultimately direct materials discovery towards improved substrates for

8 hPSC culture.

9 The topography and elastic modulus of synthetic substrates has been shown to 10 influence the differentiation of stem cells ${ }^{63-65}$. These studies highlight the importance 11 of physical as well as chemical properties in regulating self-renewal and 12 differentiation in future culture systems used for large scale manufacture of hPSCs. 13 Recently, the heparin-binding peptide GKKQRFRHRNRKG (see earlier discussion of 14 this peptide) was attached to hydrogel-based surfaces of various moduli to enable 15 mechanical control of hPSC self-renewal ${ }^{67}$. Immobilization of the peptide on the 16 hydrogel substrates was achieved by a chemoselective reaction between maleimide 17 moieties and the peptide terminated with a cysteine residue. Only stiff hydrogels (10 $18 \mathrm{kPa}$ ) were able to maintain hPSC pluripotency, which was facilitated by the activation 19 of the Yes-Associated Protein (YAP) and transcriptional coactivator with PDZ20 binding motif (TAZ). The YAP/TAZ are implicated in hPSC pluripotency and the 21 hydrogel exhibiting a Youngs Modulus of $10 \mathrm{kPa}$, determined by force indentation 22 measurements using AFM, was most effective at inducing YAP/TAZ nuclear 23 localization. 
3 To support experimental materials discovery, computational methods of predicting the

4 role of materials in encouraging cellular attachment have been explored ${ }^{68}$ (Figure 2).

5 An early example of this was demonstrated for fibroblasts by Brocchini et al. who

6 identified polymer-cell response relationships within a combinatorial library of pre-

7 synthesized copolymers spotted to form a micro array ${ }^{69}$. Linear correlations were

8 observed between fibroblast proliferation and polymer surface hydrophobicity for a

9 subset of the library of polymers. More recently human embryoid body cell adhesion

10 to a library of polymers has been linked to molecular descriptors indicating that

11 computational approached may be used to guide the design of materials production

12 for experimentation with stem cells (Figure 2). ${ }^{70}$

(a)

13

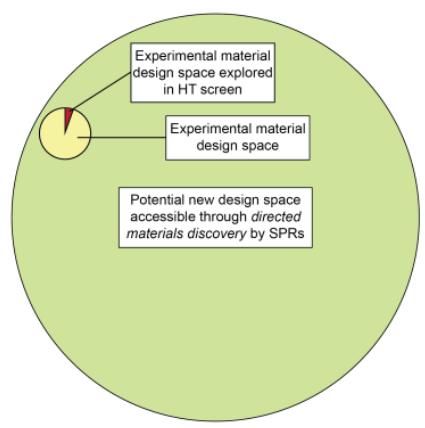

(b)

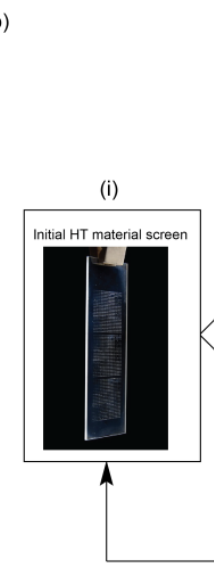

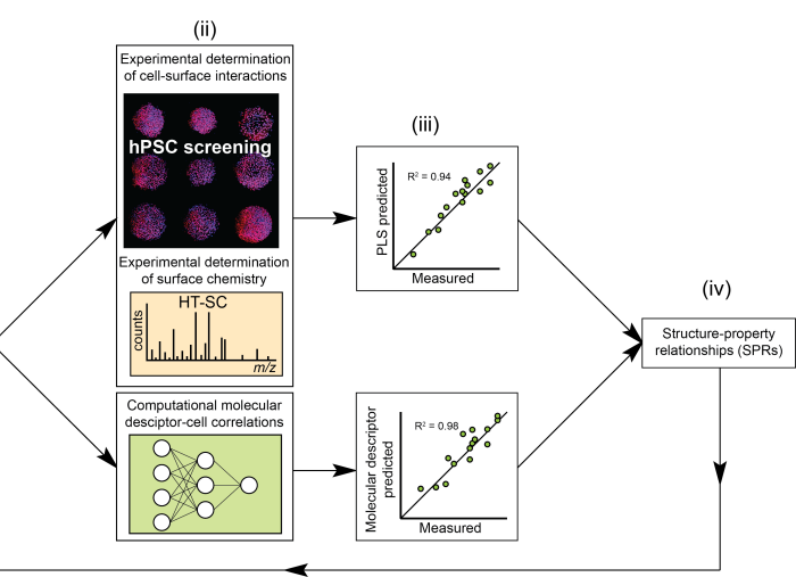

Figure 2 | High throughput materials discovery. (a) Concept: The small

15 experimental material design space that can readily be accessed limits the discovery of new materials for hPSC culture. Directed materials discovery can be achieved through advanced modelling methods that enable structure-property relationships (SPRs) to be developed. This has the potential to allow access to materials design space yet to be explored experimentally. formats such as polymer microarrays can be screened for new growth substrates for 
1 hPSC culture to access a small proportion of the potential chemical space. (ii) High

2 throughput surface characterization can be employed in parallel to the biological

3 assay. Correlation of the surface analytical data with cell performance using

4 multivariate partial least-squares (PLS) regression links structure to function. Neural

5 networks can identify molecular descriptors correlating with cell response. (iii) The

6 predictive models have the potential to identify material chemistries that could not

7 have been predicted from the experimental data alone. (iv) These advanced modelling

8 techniques can be used to develop SPRs that can be used to explore new materials

9 design space. This process can be performed in an iterative manner until an optimized 10 material has been found.

12 Surface chemical measurements can be used to determine the surface chemistries 13 controlling cell attachment to materials with the aid of statistical and machine learning 14 methods employed to aid interpretation of the large data sets. For example, Chilkoti 15 et al. characterized 15 oxygen-containing plasma-deposited films using static 16 secondary ion mass spectrometry $\left(\mathrm{SIMS}^{71}\right.$. A correlation between the positive and 17 negative ion spectra from the materials and endothelial cell growth was determined 18 using multivariate partial least-squares (PLS) regression. The PLS model identified 19 ions within the SIMS spectra that contributed towards high and low cell attachment. 20 Using a related approach, Urquhart et al. employed a combination of high throughput 21 surface characterization (surface mass spectral data) and multivariate analysis (MVA) 22 to predict the wettability of 576 polymers in a combinatorial microarray library ${ }^{72-73}$. A

23 statistically valid PLS model between water contact angle (WCA) measurements and 24 spectra obtained using time-of-flight secondary ion mass spectrometry (ToF-SIMS) 25 was obtained. This study demonstrated the utility of MVA techniques such as PLS to 
1 model datasets containing a large number of variables such as ToF-SIMS spectra

2 (each with hundreds of secondary ions) of a polymer microarray (containing hundreds

3 of materials). Following this, Mei et al. employed PLS regression to explore the

4 relationship between surface chemistry of a combinatorial polymer microarray and the

5 colony-formation frequency of hPSCs $^{53}$. Good agreement between the measured

6 colony-formation frequency and that predicted from the ToF-SIMS spectra from the

7 material surfaces was found, highlighting the importance of surface chemistry of a

8 material. The approach also helped identify the controlling surface functionalities, to

9 allow improvements from one generation to the next, to obtain the best performing

10 materials from the library of monomers available. The lead polymers were pre-

11 adsorbed with vitronectin before cell seeding. To investigate the role of this step,

12 ToF-SIMS of these protein conditioned polymer surfaces was used to analyze the

13 chemistry of this surface. Strong correlations between cell attachment and protein

14 fragment secondary ions were identified, indicating the synergy existing between the

15 material surface chemistry and the identity and amount of the adsorbed proteins to

16 enable colony-formation. It is known from blocking experiments that the role of

17 protein adsorption to polymeric growth substrates is pivotal to facilitate hPSC

18 attachment and self-renewal via engagement with cell surface integrins ${ }^{53}$.

19 Understanding which proteins adsorb from complex protein containing media to

20 materials and their conformation is an essential component in interpreting material

21 performance in protein containing culture conditions and will ultimately lead

22 materials discovery towards better substrates for hPSC culture (Box 2). Although, at

23 this time, unequivocal identification of protein identity and conformation from

24 complex protein containing media is not possible. 


\section{Box 2 $\mid$ Challenges in hPSC culture for material scientists.}

The move towards defined synthetic growth substrates for hPSC culture has been approached in a concerted manner and primarily driven by biologists aiming to achieve greater reproducibility during cell culture and remove xenogenic components. Materials scientists play an important role in meeting challenges posed by biologists via the design of growth substrates capable of achieving high expansion rates of hPSCs whilst maintaining full pluripotency potential and a normal karyotype.

A major challenge in material design is the development of a fully synthetic growth substrate that can adsorb essential proteins in the desired conformation from the culture medium to facilitate hPSC adhesion and expansion. The mechanism of hPSC adhesion to biologically inspired growth substrates that display surface moieties such as RGDcontaining peptides is well understood. However, there is gap in knowledge of how biomolecules adsorb to synthetic substrates which is limiting the rational design of improved synthetic growth substrates.

One materials design approach towards functional synthetic growth substrates is to develop synthetic mimics of biological motifs known to be beneficial for hPSC adhesion eg. employing sulfonated synthetic polymers to mimic the functional characteristics of heparin ${ }^{47}$. This hypothesis-driven approach has led to the development of polymerbased growth substrates that can achieve hPSC expansion in defined media ${ }^{47-48,53,55}$.
Another design route for synthetic growth substrates is a combination of high-throughput material and computational screening. It is often not appreciated how vast the possible 'space' of materials that could be synthesized is, and that it is not possible to explore even a tiny fraction of this by experiments. The vast design space of synthetic biomaterials presents many opportunities to discover better synthetic growth substrates. To meet this challenge and explore the chemical combinatorial design space more effectively; computational methods will be needed to complement experimentally derived hypotheses and better inform materials discovery screening. Computational modelling can contribute in several ways. Use of design of experiments (DoE) methods allows the number of experiments that are required to cover a given design space to be minimized. Computational models of the data from these experiments allow the properties of all materials within the design space to be predicted.

If large-scale high throughput methods can be developed that capture sufficient molecular diversity on the materials, these models are capable of wide extrapolation into materials space. Finally, evolutionary methods are beginning to be applied to materials design and discovery. They allow initial sets of promising materials to be evolved towards a desired materials property 'fitness function' in an experimentally efficient way. These methods are among the most efficient at exploring extremely large design spaces. (Figure 2). 


\section{Outlook}

3 Human pluripotent stem cells (hPSCs) have present possibilities in a wide variety of

4 applications such as regenerative medicine and pharmaceutical drug screening. In the

5 future, stem cell factories will be required to produce the large numbers of hPSC that

6 will be required to meet the demand for billions of cells typical of regenerative

7 medicine interventions currently in clinical trials. The long-term expansion and self-

8 renewal of hPSCs in xeno- and defined conditions is a prerequisite to achieving this.

9 Xeno- and feeder-free E8 medium has now been commercialized ${ }^{21}$ and E6, a medium

10 used to reprogram somatic cells to hiPSCs prior to expansion in E8, is currently under

11 development. For adherent hPSC culture this will also need to be supported by 12 chemically defined substrates that offer high growth rates in a reproducible manner.

13 Suspension hPSC culture has been shown as a promising alternative to adherent hPSC

14 culture. However, refinement of the culture media components and improved growth 15 rates versus adherent systems will need to be demonstrated before suspension culture 16 can be considered as a viable alternative.

18 The most recently developed defined substrates are polymers and peptides that are 19 applicable to a xeno- and serum-free environment but few are able to support the 20 expansion of hPSCs at levels similar to that of the current gold standard (but 21 undefined) growth substrates, such as Matrigel. A number of novel polymers show 22 promise, but still largely require protein pre-adsorption or display significant 23 limitations in industrial scale up-mainly cost. 
1 The search for new materials for adherent hPSC culture has been greatly accelerated

2 by the recent application of high throughput sample screening strategies such as

3 polymer microarrays. Surface characterization and correlative and predictive models

4 make this a powerful approach with which to search for new materials. Development

5 of quantitative structure-property relationships (SPRs) using the results from large

6 experimental libraries linking polymer structure to hPSC performance on materials

7 are likely to broaden the chemical combinatorial space beyond what is currently

8 explored to facilitate the search for better materials for hPSC culture. The materials

9 discovery process can be further aided through combination with high throughput

10 screening of synthetic soluble factors that can replace biologically derived ingredients

11 within hPSC culture media and increase the scalability of such culture systems.

13 High throughput materials screening is moving towards the ability to carry out

14 directed high throughput materials discovery to allow exploration beyond the existing 15 experimentally investigated chemical space utilizing experimentally determined 16 surface structure-property relationships (sSPRs) and computationally determined 17 molecular descriptors. This will represent a step change in materials discovery 18 capabilities to enable these discovery methods to evolve beyond the constraints of the 19 current experimentally accessible chemical space. Recently, molecular descriptors 20 have been used to predict the response of stem cell attachment and generate sSPRs in 21 silicio, without the need for experimentally determined polymer characteristics ${ }^{70}$.

22 Although the "reverse SPR" problem (backing out a polymer structure from an SPR 23 model) has been challenging in the past, exciting new developments in mathematics 24 have recently provided practical methods for designing polymers with optimal 25 properties from structure-property relationships and molecular descriptors ${ }^{74}$. Success 
1 in this endeavor would open up the full range of materials to computational

2 examination to direct synthesis efforts to potentially fruitful areas for experimental

3 exploration. We anticipate that these and the other material discovery approaches

4 covered in this review will provide the new materials necessary for the stem cell

5 factories of the future required to supply emerging stem cell therapies.

6

\section{References}

1. Kohn, J. New approaches to biomaterials design. Nature Mater. 3, 745-747 (2004).

2. Place, E. S., Evans, N. D. \& Stevens M. M. Complexity in biomaterials for tissue engineering. Nature Mater. 8, 457-470 (2009).

3. Hook, A. L. et al. High throughput methods applied in biomaterials development and discovery. Biomaterials 31, 187-198 (2010).

4. de Boer, J. \& van Blitterswijk, C. Materiomics: High-Throughput Screening of Biomaterial Properties. (Cambridge Univ. Press, Cambridge, 2013)

5. Thomson, J. A. et al. Embryonic stem cell lines derived from human blastocytes. Science 282, 1145-1147 (1998).

6. Takahashi, K. \& Yamanaka, S. Induction of pluripotent stem cells from mouse embryonic and adult fibroblast cultures by defined factors. Cell 126, 663-676 (2006).

7. Rajamohan, D., Matsa, E. \& Kalra S. Current status of drug screening and disease modelling in human pluripotent stem cells. BioEssays 35, 281-298 (2012).

8. Thomas, R. J. et al. Automated, scalable culture of human embryonic stem cells in feeder-free conditions. Biotech. Bioeng. 102, 1636-1644 (2009).

9. Mahlstedt, M. M. et al. Maintenance of pluripotency in human embryonic stem cells cultured on a synthetic substrate in conditioned medium. Biotech. Bioeng. 105, 130-140 (2010).

10. Xu, C. et al. Feeder-free growth of undifferentiated human embryonic stem cells. Nature Biotech. 19, 971-974 (2001).

11. Martin, M. J., Muotri, A., Gage, F. \& Varki, A. Human embryonic stem cells express an immunogenic nonhuman sialic acid. Nature Med. 11, 228-232 (2005). 
12. Kleinman, H. K. et al. Isolation and characterization of type-IV procollagen, laminin, and heparin-sulfate proteoglycans from EHS sarcoma. Biochem. 21, 6188-6193 (1982).

13. Jin, S., Yao, H., Weber, J. L., Melkoumian, Z. K. \& Ye, K. A synthetic, xenofree peptide surface for expansion and directed differentiation of human induced pluripotent stem cells. PLOS ONE 7:11 (2012).

14. Nagaoka, M., Si-Tayeb, K., Akaike, T. \& Duncan, S. A. Culture of human pluripotent stem cells using completely defined conditions on a recombinant E-cadherin substratum. BMC Dev. Bio. 10:60 (2010).

15. Stelzer, T., Marwood, T. \& Neeley, C. Innovative animal component-free surface for the cultivation of human embryonic stem cells. BMC Proc. 5(Suppl 8):P51 (2011)

16. Swistowski, A. et al. Xeno-free defined conditions for culture of human embryonic stem cells, neural stem cells and dopaminergic neurons derived from them. PLOS ONE 4:7 (2009)

17. Ludwig, T. E. et al. Derivation of human embryonic stem cells in defined conditions. Nature Biotech. 24, 185-187 (2006).

18. Wang, L. et al. Self-renewal of human embryonic stem cells requires insulinlike growth factor-1 receptor and ERBB2 receptor signalling. Blood 110, 4111-4119 (2006).

19. Bergstrom, R., Strom, S., Holm, F., Feki, A. \& Hovatta, O. Xeno-free culture of human pluripotent stem cells. Methods Mol. Biol. 767, 125-136 (2011).

20. Chen, G. et al. Chemically defined conditions for human iPSC derivation and culture. Nature Meth. 8, 424-429 (2011).

21. Li, Y., Powell, S., Brunette, E., Lebkowski, J. \& Mandalam, R. Expansion of human embryonic stem cells in defined serum-free medium devoid of animalderived products. Biotech. Bioeng. 91, 688-698 (2005).

22. Genbacev, O. et al. Serum-free derivation of human embryonic stem cell lines on human placental fibroblast feeders. Fertil. Steril. 83, 1517-1529 (2005).

23. Rajala, K. et al. A defined and xeno-free culture method enabling the establishment of clinical-grade human embryonic, induced pluripotent and adipose stem cells. PLOS ONE 5:4 (2010).

24. Furue, M. K. et al. Heparin promotes the growth of human embryonic stem cells in a defined serum-free medium. PNAS 105, 13409-13414 (2008). 
25. Amit, M., Shakiri, C., Margulets, V. \& Itskovitz-Eldor, J. Feeder layer- and serum-free culture of human embryonic stem cells. Biol. Reprod. 70, 837-845 (2004).

26. Watanabe, K. et al. A ROCK inhibitor permits survival of dissociated human embryonic stem cells. Nature Biotech. 25, 681-686 (2007).

27. $\mathrm{Xu}, \mathrm{Y}$. et al. Revealing a core signalling regulatory mechanism for pluripotent stem cell survival and self-renewal survival by small molecules. PNAS 107, 8129-8134 (2010).

28. Tsutsui, H. et al. An optimized small molecule inhibitor cocktail supports long-term maintenance of human embryonic stem cells. Nat Commun 2, 167 (2011).

29. Qi, X. et al. BMP4 supports self-renewal of embryonic stem cells by inhibiting mitogen-activated protein kinase pathways. Proc Natl Acad Sci U S A 101, 6027-6032 (2004).

30. Damoiseaux, R., Sherman, S.P., Alva, J.A., Peterson, C. \& Pyle, A.D. Integrated chemical genomics reveals modifiers of survival in human embryonic stem cells. Stem Cells 27, 533-542 (2009).

31. Barbaric, I. et al. Novel regulators of stem cell fates identified by a multivariate phenotype screen of small compounds on human embryonic stem cell colonies. Stem Cell Res 5, 104-119 (2010).

32. Buehr, M. et al. Capture of authentic embryonic stem cells from rat blastocysts. Cell 135, 1287-1298 (2008).

33. Cai, J. et al. Assessing self-renewal and differentiation in human embryonic stem cell lines. Stem Cells 24, 516-530 (2006).

34. Sato, N., Meijer, L., Skaltsounis, L., Greengard, P. \& Brivanlou, A.H. Maintenance of pluripotency in human and mouse embryonic stem cells through activation of Wnt signaling by a pharmacological GSK-3-specific inhibitor. Nat Med 10, 55-63 (2004).

35. Bone, H.K. et al. Involvement of GSK-3 in regulation of murine embryonic stem cell self-renewal revealed by a series of bisindolylmaleimides. Chem Biol 16, 15-27 (2009).

36. Xiong, L. et al. Heat shock protein 90 is involved in regulation of hypoxiadriven proliferation of embryonic neural stem/progenitor cells. Cell Stress Chaperones 14, 183-192 (2009).

37. Miyabayashi, T., Yamamoto, M., Sato, A., Sakano, S. \& Takahashi, Y. Indole derivatives sustain embryonic stem cell self-renewal in long-term culture. Biosci Biotechnol Biochem 72, 1242-1248 (2008). 
38. Chen, L. \& Khillan, J.S. Promotion of feeder-independent self-renewal of embryonic stem cells by retinol (vitamin A). Stem Cells 26, 1858-1864 (2008).

39. Li, M. et al. Neuronal differentiation of C17.2 neural stem cells induced by a natural flavonoid, baicalin. Chembiochem 12, 449-456 (2011).

40. Anneren, C., Cowan, C.A. \& Melton, D.A. The Src family of tyrosine kinases is important for embryonic stem cell self-renewal. J Biol Chem 279, 3159031598 (2004).

41. Miyazaki, T. et al. Recombinant human laminin isoforms can support the undifferentiated growth of human embryonic stem cells. Biochem. Biophys. Res. Comm. 375, 27-32 (2008).

42. Miyazaki, T. et al. Laminin E8 fragments support efficient adhesion and expansion of dissociated human pluripotent stem cells. Nature Commun. 3, 1236-1245 (2012).

43. Rodin, S. et al. Long-term self-renewal of human pluripotent stem cells on human recombinant laminin-511. Nature Biotech. 28, 611-615 (2010).

44. Derda, R. et al. Defined substrates for human embryonic stem cell growth identified from surface arrays. ACS Chem. Bio. 2, 347-355 (2007).

45. Melkoumian, Z. et al. Synthetic peptide-acrylate surfaces for the long-term self-renewal and cardiomyocyte differentiation of human embryonic stem cells. Nature. Biotech. 28, 606-610 (2010).

46. Brafman, D. A. et al. Long-term human pluripotent stem cell self-renewal on synthetic polymer surfaces. Biomaterials 31, 9135-9144 (2010).

47. Villa-Diaz, L. G. et al. Synthetic polymer coatings for long-term growth of human embryonic stem cells. Nature Biotech. 28, 581-583 (2010).

48. Irwin, E. E., Gupta, R., Dashti, D. C. \& Healy, K. E. Engineered polymermedia interfaces for the long-term self-renewal of human embryonic stem cells. Biomaterials 32, 6912-6919 (2011).

49. Klim, J. R., Li, L. Y., Wrighton, P. J., Piekarczyk, M. S. \& Kiessling L. L. A defined glycosaminoglycan-binding substratum for human pluripotent stem cells. Nature Meth. 7, 989-996 (2010).

50. Anderson, D. G., Putnam, D., Lavik, E. B., Mahmood, T. A. \& Langer, R. Biomaterial microarrays: rapid, microscale screening of polymer-cell interaction. Biomaterials 26, $4892-4897$ (2005).

51. Brocchini, S., James, K., Tangpasuthadol, V. \& Kohn, J. A combinatorial approach for polymer design. JACS 119, 4553-4554 (1997). 
52. Anderson, D. G., Levenburg, S. \& Langer, R. Nanoliter-scale synthesis of arrayed biomaterials and application to human embryonic stem cells. Nature Biotech. 22, 863-866 (2004).

53. Mei, Y. et al. Combinatorial development of biomaterials for clonal growth of human pluripotent stem cells. Nature Mater. 9, 768-778 (2010).

54. Saha, K. et al. Surface-engineered substrates for improved pluripotent stem cell culture under fully defined conditions. PNAS 108, 18714-18719 (2011).

55. Zhang, R. et al. A thermoresponsive and chemically defined hydrogel for long-term culture of human embryonic stem cells. Nature Commun. 4, 13351345 (2013).

56. Meng, Y. et al. Characterization of integrin engagement during defined human embryonic stem cell culture. Faseb Journal 24, 1056-1065 (2010).

57. Harb, N., Archer, T. \& Sato, N. The Rho-ROCK-Myosin axis determines cellcell integrity of self-renewing pluripotent stem cells. PLOS ONE 3:8 (2008).

58. Rowland, T. J. et al. Roles of integrins in human induced pluripotent stem cell growth on Matrigel and vitronectin. Stem Cells Dev. 19, 1231-1240 (2010).

59. Prowse, A. B. J., Chong, C., Gray, P. P. \& Munro, T. P. Stem cell integrins: implications for ex-vivo culture and cellular therapies. Stem Cell Res. 6, 1-12 (2011).

60. Li, L., Bennett, S. A. L. \& Wang, L. Role of E-cadherin and other cell adhesion molecules in survival and differentiation of human pluripotent stem cells. Cell Adh. Migr. 6, 59-70 (2012).

61. Koenig, A. L., Gambillara, V. \& Grainger, D. W. Correlating fibronectin adsorption with endothelial cell adhesion and signalling on polymer substrates. J. Biomed. Mater. Res. Part A 64, 20-37 (2003).

62. Weber, N., Bolikal, D., Bourke, S. L. \& Kohn, J. Small changes in the polymer structure influence the adsorption behaviour of fibrinogen on polymer surfaces: validation of a new rapid screening technique. J. Biomed. Mater. Res. Part A. 68, 496-503 (2004).

63. Ingber, D. E. The riddle of morphogenesis: a question of solution chemistry or molecular cell engineering? Cell 75, 1249-1252 (1993).

64. Wan, L. Q. et al. Geometric control of human stem cell morphology and differentiation. Integr. Biol. 2, 346-353 (2010).

65. Fu, J. et al. Mechanical regulation of cell function with geometrically modulated elastomeric substrates. Nature Meth. 7, 733-736 (2010). 
66. Trappmann, B. et al. Extracellular-matrix tethering regulates stem-cell fate. Nature Mater. 27, 642-649 (2012).

67. Musah, S. et al. Glycosaminoglycan-binding hydrogels enable mechanical control of human pluripotent stem cell self-renewal. ACS Nano 6, 1016810177 (2012).

68. Cranford, S. W., de Boer, J., van Blitterswijk, C., Buehler, M. J. Materiomics: An -omics approach to biomaterials research. Adv. Mater. 25, 802-824 (2013).

69. Brocchini, S., James, K., Tangpasuthadol, V. \& Kohn, J. Structure-property correlations in a combinatorial library of biodegradable materials. J. Biomed. Mater. Res. 42, 67-75 (1998).

70. Epa, V. C. et al. Modelling human embryoid body cell adhesion to a combinatorial library of polymer surfaces J. Mater. Chem. 22, 20902-20906 (2012).

71. Chilkoti, A., Schmierer, A. E., Pérez-Luna, V. H. \& Ratner, B. D. Investigating the relationship between surface chemistry and endothelial cell growth: partial least-squares regression of the static secondary ion mass spectra of oxygen-containing plasma-deposited films. Anal. Chem. 67, 28832891 (1995).

72. Urquhart, A. J. et al. High throughput surface characterisation of a combinatorial material library. Adv. Mater. 19, 2486-2491 (2007).

73. Urquhart, A. J. et al. TOF-SIMS analysis of a 576 micropatterned copolymer array to reveal surface chemical moieties that control wettability. Anal. Chem. 80, 135-142 (2008).

74. Martin S. Lattice enumeration for inverse molecular design using the signature descriptor. J. Chem. Inf. Model. 52, 1787-1797 (2012). 